\title{
IMAGING TECHNIQUES USED IN MULTIPLE MYELOMA
}

\author{
Abdul Hameed ${ }^{1}$, Paul Foran'2, Jonathan Ryan², Peter O'Gorman ${ }^{3}$ \\ ${ }^{1}$ Department of Medical Oncology, Shaukat Khanum Memorial Cancer Hospital and Research Centre, Lahore, \\ Pakistan, ${ }^{2}$ Department of Radiology, Mater Misericordiae University Hospital, Dublin, Ireland, ${ }^{3}$ Department of \\ Haematology, Mater Misericordiae University Hospital, Dublin, Ireland \\ Received: 19 March 2015 / Accepted: 20 April 2015
}

\begin{abstract}
Multiple myeloma (MM) is a plasma cell disorder, characterised by bone marrow infiltration with clonal plasma cells; production of monoclonal immunoglobulin (paraprotein); end-organ damage; lytic lesions in the bones; renal impairment; hypercalcaemia and anaemia. Skeleton evaluation in MM is necessary not only for staging purposes but also to detect serious complications such as fractures. Skeletal survey is an established first-line investigation for this purpose. However, in recent years, new imaging techniques such as whole-body magnetic resonance imaging and 2-fluoro-2-deoxy-D-glucose positron emission tomography-computed tomography have been used widely. In this article, we review different imaging techniques used in $\mathrm{MM}$ and their impact on patient management.
\end{abstract}

Key words: Imaging techniques, magnetic resonance imaging, multiple myeloma, osteolytic lesions, positron emission tomography/computed tomography, skeletal survey

\section{Introduction}

Multiple myeloma (MM) is a plasma cell malignancy. The aetiology of MM is unknown and it accounts for $10 \%$ of haematological malignancies and nearly $1 \%$ of all cancers. ${ }^{[1]}$ Median age at presentation is 70 years and only $2-3 \%$ of patients are younger than 40 years. MM has slight male predominance and is more common among African Americans than white Americans or Europeans. ${ }^{[2]}$ MM is characterised by bone marrow (BM) infiltration with clonal plasma cells, originating from B-cells and production of immunoglobulins (paraprotein) that can be detected in serum or urine by electrophoresis and/or immunofixation. The paraproteins secreted in MM are $\operatorname{IgG}(52 \%), \operatorname{IgA}(21 \%)$ and only light chains $(16 \%)$ and about $2 \%$ of cases are non-secretary. A majority of patients (about 80\%) have osteolytic bone lesions (OBL) at diagnosis. Other presenting features are anaemia $(72 \%)$, hypercalcaemia (13\%), renal impairment $(19 \%)$ and recurrent infections. $20 \%$ of cases are asymptomatic. ${ }^{[3,4]}$ BM biopsy, biochemistry and comprehensive evaluation of skeleton are vital in the diagnosis of $\mathrm{MM}$ and to differentiate it from other plasma cell disorders [Figure 1].

Correspondence: Dr. Abdul Hameed, Department of Medical Oncology, Shaukat Khanum Memorial Cancer Hospital and Research Centre, Lahore, Pakistan. Email: abdulh@skm.org.pk
The minimum criteria for making a diagnosis of $\mathrm{MM}$ include BM plasma cells $>10 \%$, detection of monoclonal proteins in serum or urine and the presence of end-organ damage. This group of symptoms is abbreviated as CRAB: Hypercalcaemia, renal impairment, anaemia and bony lesions.

\section{Myeloma Bone Disease (MBD)}

Nearly $90 \%$ of MM patients suffer from MBD during the course of the disease. Increased osteoclastic activity and suppressed osteoblastic function are a key factor in the pathophysiology of MBD. ${ }^{[5,6]}$ Classically, these are punched out lytic lesions [Figure 2] without surrounding sclerosis around due to the absence of anabolic activity. Osteolytic lesions are located in close vicinity of plasma cells promoting the notion of interaction between myeloma cells and BM stromal cells leading to release of certain chemokines resulting in upregulation of osteoclastic and downregulation of osteoblastic activity. These lesions arise from medulla and move outward causing endosteal scalloping of the cortex and sometimes invading the periosteum leading to the formation of extraosseous mass. ${ }^{[6]}$ Other types of lesions are solitary plasmactyomas and in about $10-15 \%$ of cases, there is a generalised osteopenia or osteoporosis. The axial 


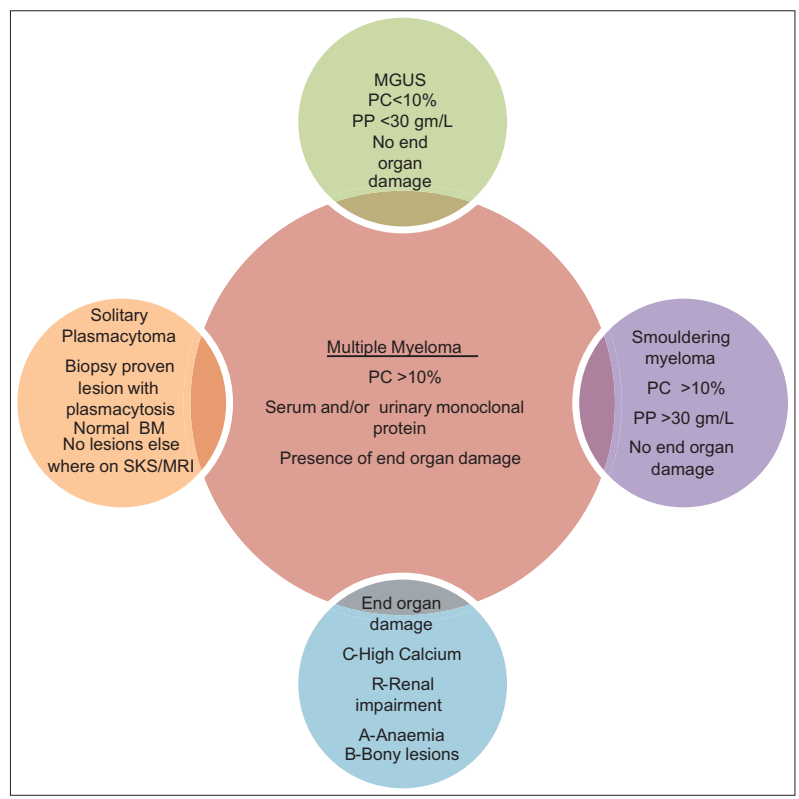

Figure 1: Diagnostic criteria for multiple myeloma and related disorders 5. PC: Plasma cells, PP: Paraprotein

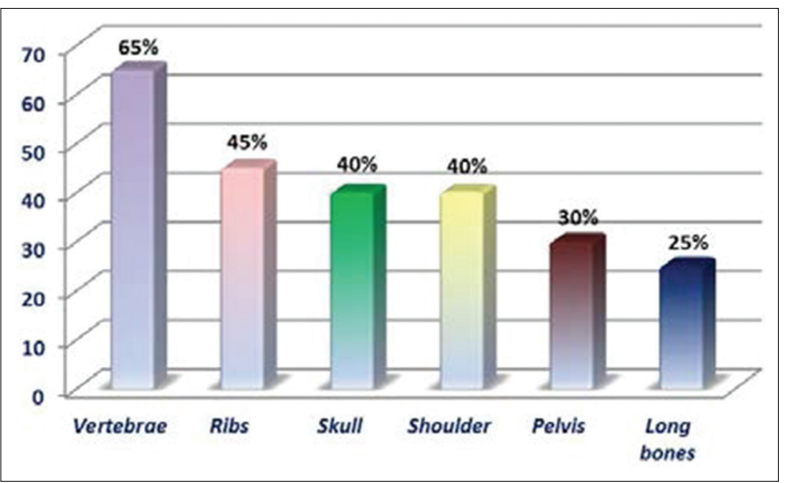

Figure 2: Plain radiographs of (a) skull, (b) humerus and (c) femora showing multiple lytic lesions

skeleton comprising the vertebrae, ribs, skull, and pelvis, along with the proximal parts of long bones are most commonly involved [Figure 3]. ${ }^{[2,7]}$ Sclerotic lesions in MM can also be seen in POEMS syndrome (polyneuropathy, organomegaly, endocrinopathy, monoclonal gammopathy and skin changes) which accounts for $<1 \%$ of MM patients. ${ }^{[8]}$ Osteolytic lesions rarely heal and they are persistent even when the disease is in remission. However, with the use of novel treatments, there is some evidence that certain agents such as bortezomib and increase osteoblastic activity in MM patients. ${ }^{[9]}$

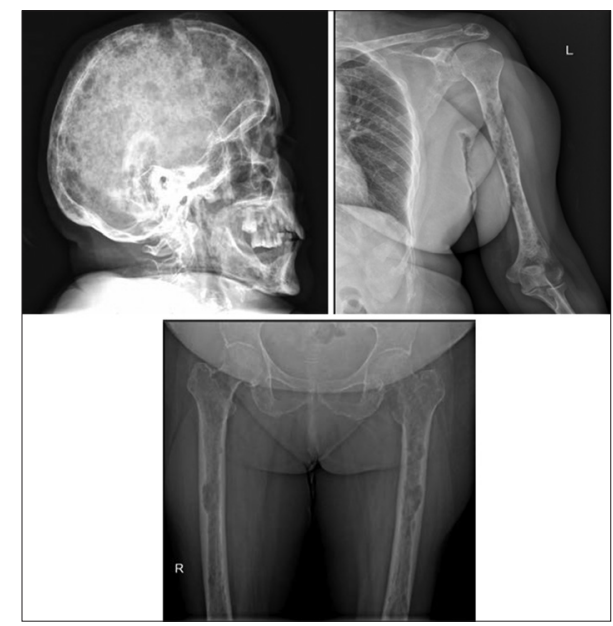

Figure 3: Skeletal site involvement in myeloma bone disease. Percentages mentioned are not meant to add up to $100 \%$ as most patients have multi-site involvement

Imaging has a key role in the assessment of disease at the time of diagnosis and subsequently for the monitoring of response to therapy. Imaging tools also highlight the presence or absence of any extramedullary disease and detect complications resulting from bone disease, for example, fractures. Conventional radiography (SKS) and new imaging modalities are used for the above purposes. All imaging techniques used have some advantages and disadvantages and might be appropriate for certain subgroups of MM.

\section{Plain Radiography (Skeletal Survey [SKS])}

SKS still remains the primary investigation for initial workup of MM, particularly where other modern facilities of imaging are unavailable. SKS includes about 20 plain films, taken as frontal (AP) and lateral (LA) views of chest, skull, whole spine, pelvis, humeri and femora. It covers a large area of the skeleton and highlights common complications of MM including lytic lesions, osteoporosis and fractures [Figure 2]..$^{[2,8]}$ It is readily available and cheap and has a low radiation exposure. Cortical bone lesions are better defined on plain radiograph compared to magnetic resonance imaging (MRI) ${ }^{[4]}$ However, SKS has a high false negative rate $(30-70 \%)$, resulting in an underestimation of the stage and the diagnosis. The lytic lesions appear on radiographs when $30-75 \%$ of cancellous bone is lost, meaning lesions may not be picked up at an early disease stage. ${ }^{[2,10]}$ 
SKS provides limited information about ribs, sternum and scapulae. Furthermore, it is time-consuming with regular posture changes which could be painful for some patients who may already have fractures. In addition, it is difficult on SKS to differentiate between osteopenia due to $\mathrm{MM}$ and that due to other common causes such as postmenopause or senile osteoporosis. ${ }^{[11]}$ As lytic lesions rarely heal, SKS does not help in the assessment of response to therapy [Table 1].

\section{Computed Tomography (CT)}

CT is another modality used in MM for the evaluation of bony lytic lesions [Figure 4]. Whole body CT was introduced to evaluate the whole skeleton. In some institutions, CT is used as initial imaging for the assessment of the spine and pelvis. It is an ideal investigation for the detection of early bone destruction. Extramedullary lesions, soft tissue mass, diffuse osteopenia, fractures and rare osteosclerotic lesions can also be detected on CT with

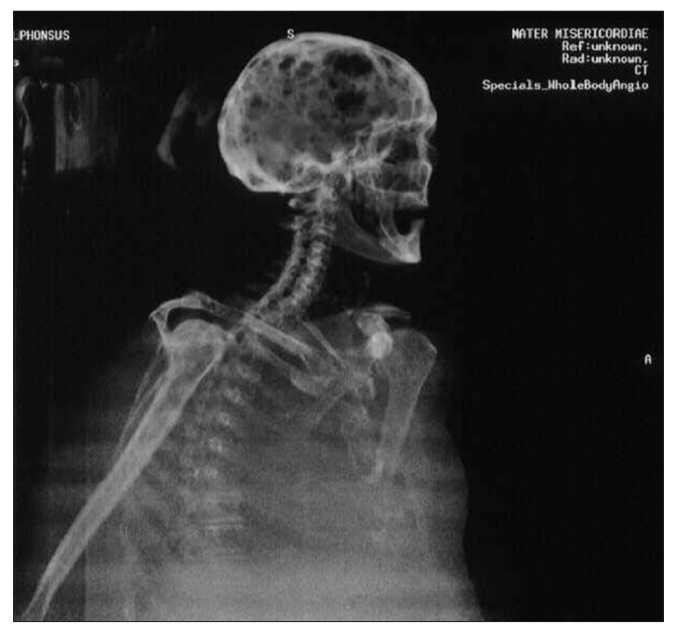

Figure 4: Whole-body computed tomography of a patient with multiple myeloma showing multiple lytic lesions higher sensitivity compared to SKS. Furthermore, it has been reported that $\mathrm{CT}$ reveals more lesions in areas that are poorly visualised on plain radiography. CT is superior in the assessment of fracture risk in unstable areas compared to both plain radiography and MRI. ${ }^{[12,13]} \mathrm{CT}$ is faster and the patient does not need to change postures as in plain radiography and there is no need of contrast media, for skeletal imaging which could be dangerous in MM.

Patients who may already have compromised renal function due to the primary disease. Moreover, multidetector row CT (MDCT), a novel CT technique, is quite sensitive in the detection of smaller lytic lesions $(<5 \mathrm{~mm})$ particularly in the spine area. In addition, with this technique, there is a clear distinction between bony structures and soft tissues leading to less false positive results. ${ }^{[13,14]}$ Another advantage of $\mathrm{CT}$ is its accuracy in a demonstration of extraosseous lesions and is a tool of choice for performing guided biopsy from spine or pelvis [Table 2]. CT is also used for planning of radiotherapy. CT can also be used in the detection of spinal cord compression; however, MRI is the preferred imaging modality in such patients.

When compared to plain radiography, CT carries a high radiation exposure (1.3-3 times higher $)^{[11]}$ and like conventional X-rays, is non-specific in the assessment of osteopenia. There is a relatively high false negative rate, particularly in cases of diffuse marrow infiltration and in early stages of the disease when there is no bone destruction. ${ }^{[15]}$

\section{Whole-body low-dose CT (WBLDCT)}

Due to high radiation exposure associated with whole-body CT, WBLDCT was introduced to overcome this drawback. The effective radiation dose in WBLDCT is much lower

Table 1: Advantages and limitations of skeletal survey

\begin{tabular}{l|l}
\hline Advantages & Limitations \\
\hline Readily available & Low sensitivity (47.4\%) \\
\hline Inexpensive & $10-20 \%$ lesions are missed \\
\hline Detection of lytic lesions, fractures and osteoporosis & $\begin{array}{l}\text { Early stage lesions are missed (>30\% cortical bone loss should be } \\
\text { present before lytic lesions appear) }\end{array}$ \\
\hline \multirow{2}{*}{ Low radiation exposure } & Poor tolerability (20 exposures, changing posture and elderly patients) \\
\cline { 2 - 2 } & Not useful in the evaluation of treatment response as lesions rarely heal \\
\cline { 2 - 2 } & Limited evaluation of certain areas, such as scapulae, sternum and ribs \\
\hline
\end{tabular}


Table 2: Role of computed tomography in multiple myeloma

\begin{tabular}{l|l}
\hline Advantages & Limitations \\
\hline Detection of smaller lesions & High radiation exposure (1.3-3 times) \\
\hline Comprehensive evaluation in a short time & Non specific in cases of osteopenia \\
\hline High sensitivity (70.4\%) & High false negative rate (in diffuse or in the early phase of disease) \\
\hline Detection of extramedullary disease & Cost \\
\hline Exact location of lesions (helpful in biopsy) & Availability \\
\hline Assessment of fracture risk & \\
\hline Planning for radiotherapy & \\
\hline
\end{tabular}

compared to standard CT (3.3 millisieverts [mSv] vs. $36.6 \mathrm{mSv})$ and less than double that of SKS $(3.3[\mathrm{mSv}]$ vs. $1.7 \mathrm{mSv}){ }^{[12,13]}$ Gleeson et al. reported a high diagnostic accuracy of WBLDCT in the detection of lytic lesions compared to SKS. More than half of the cases were upstaged and WBLDCT revealed additional information, for example, lymphadenopathy, hepatosplenomegaly and lung lesions which were not seen on SKS. Moreover, WBLDCT was superior to whole-body magnetic resonance imaging (WBMRI) in detection of residual lytic lesions. ${ }^{[13]}$

In brief, standard or low dose, CT is an alternative to plain radiography in the assessment of spine lesions in patients with symptoms of pain, as it provides a more comprehensive evaluation in a short period of time. Furthermore, in patients who are symptomatic in spite of negative SKS, CT may reveal additional lesions. ${ }^{[1]}$

\section{MRI}

There are two types of marrow; the red marrow or active haematopoietic and the yellow fatty inactive marrow. The red marrow converts to yellow fatty marrow as age advances, and in adults, haematopoietic marrow is mostly localised to the axial skeleton. There are five patterns of BM infiltration seen on MRI. These are: (1) Normal marrow (low tumour burden), (2) focal lesions (FLs), (3) variegated (salt and pepper appearance), (4) diffuse disease and (5) focal and diffuse infiltration. Thus, MRI appearance in MM patients will be dependent on the status of the underlying BM (red or yellow) and pattern of involvement. ${ }^{[16,17]}$ The appearance of BM infiltration correlates with disease stage; normal and variegated pattern is consistent with Stage I, whereas focal and diffuse infiltration was associated with Stage II or III. ${ }^{[18]}$ Normal

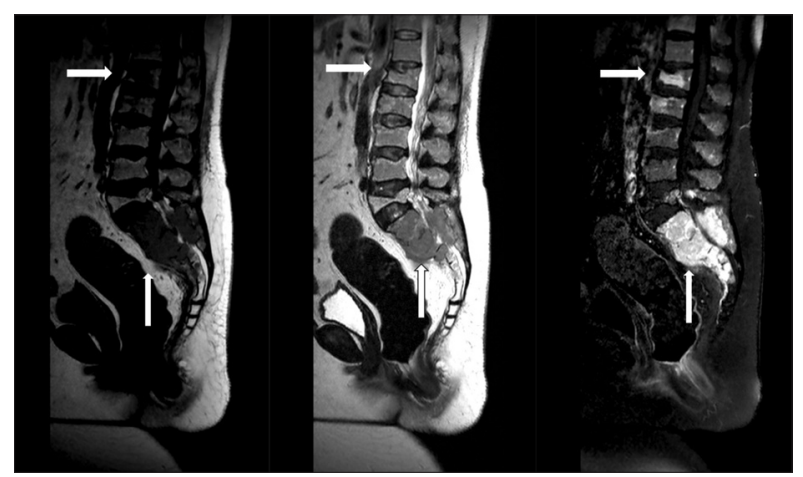

Figure 5: T1 low, T2 isointense and post gadolinium $\mathrm{T} 1$ enhancing focal vertebral lesion represents myelomatous deposits in the lumbar (white arrow) and sacral vertebral body. The associated lobulated enhancing soft tissue (red arrow) in relation to the sacral vertebral body is extending in the sacral neural foramina and compromising sacral nerve roots

BM appearances are present in about $50-75 \%$ of untreated Stage-I (according to Durie-Salmon staging system) and in $20 \%$ of untreated Stage-III disease. ${ }^{[17]}$

\section{MRI sequences}

The most common MRI sequences used in MM are T1-weighted, the T2-weighted with fat suppression, the short time inversion recovery (STIR) and the gadolinium T1-weighted with fat suppression. The classic myeloma lesions appear as low signal intensity on T1-weighted images and hyperintense on T2-weighted and STIR images. They show enhancement with gadolinium [Figure 5]. ${ }^{[19]}$

\section{Uses in MM}

MRI is ideal in the detection of BM infiltration with myeloma cells even before bone destruction is evident on plain radiographs. ${ }^{[11,20]}$ This is an investigation of choice 
in cases of suspected spinal cord compression. It provides a comprehensive assessment of the extent and exact site of compression. MRI can be helpful in the differentiation of malignant from benign lesions and the evaluation of fracture risk. MM patients with $>10$ lesions on MRI have 6-10 times higher risk of fracture compared to those who had either no or $<10$ lesions. ${ }^{[21]} \mathrm{MRI}$ has a key role in the detection of certain complications, for example, avascular necrosis of head of femur and amyloid deposits in heart or other soft tissues. ${ }^{[22]}$

\section{Role of MRI in solitary plasmacytoma/smouldering $\mathrm{MM} /$ monoclonal gammopathy of underdetermined significance (MGUS)}

MRI is also recommended in all cases of solitary bone plasmacytoma (SBP) and may reveal additional lesions which were not detected on plain radiography. In one study, MRI of spine detected additional foci in four out of 12 patients. ${ }^{[23]}$ In cases of smouldering MM (smouldering myeloma [SM]), MRI detected marrow abnormalities in $30-50 \%$ of patients. Furthermore, in one study it was demonstrated that median time to start treatment was 16 months in patients with abnormal MRI compared to 43 months with normal MRI $(P<0.01) .{ }^{[17,24]}$ Based on recent published data, the presence of two or more lesions ( $>5 \mathrm{~mm}$ ) on MRI is an indication to start therapy in these patients. ${ }^{[25]}$ There are some studies using MRI in MGUS patients. In one study, evaluating 24 patients, the thoracolumbar spine MRI was normal in all MGUS cases versus only six of $44 \mathrm{MM}$ patients who had normal findings. ${ }^{[26]}$ In another study, Berg et al. reported BM abnormalities in $19 \%(n=37)$ of cases. These patients had progression to $\mathrm{MM}$ in a shorter time compared to the cases with normal MRI. ${ }^{[27]}$ It has also been noted that MGUS patients with abnormal MRI have a higher paraprotein and plasma cell percentage in BM compared to cases with normal MRI. ${ }^{[28]}$ In brief, MRI is very helpful in cases of SBP, SM; however, in MGUS it may be useful in subgroups of patients who have a high risk of disease progression [Figure 6].

\section{WBMRI}

MRI provides valuable information about most of the axial skeleton; however, some bony areas may not be assessed properly including the skull, ribs and clavicle due to respiratory movements. To resolve this issue and

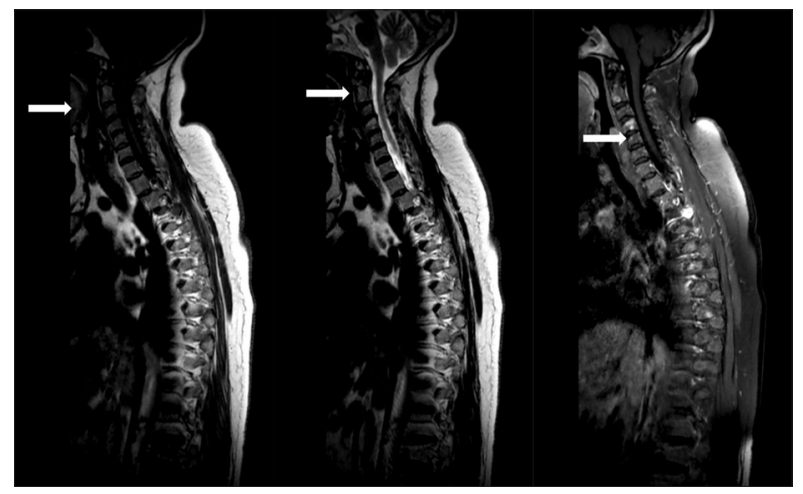

Figure 6: $\mathrm{T} 1$ low, $\mathrm{T} 2$ isointense and post gadolinium $\mathrm{T} 1$ enhancing focal $\mathrm{C} 2$ vertebral bodies shows marrow deposits (white arrow)

to have a comprehensive view, WBMRI was introduced. WB MRI detects significant BM involvement in up to $20 \%$ of cases which are negative on SKS. In cases, where both modalities were positive MR shows more extensive bone morrow infiltration. ${ }^{[29]}$ In another, large study $(n=611)$, WBMRI was compared with SKS. WBMRI revealed lesions in $74 \%$ of imaged sites compared to $43 \%$ with SKS. Moreover, in patients with normal SKS about half showed FLs on MRI. The MRI was superior to SKS, particularly in spine, pelvis and sternum. However, it was inferior to SKS in the detection of FLs in ribs and long bones. ${ }^{[30]}$

When compared to WBLDCT, WBMRI was found to be superior in both focal and diffuse disease appearances and revealed more lesions than WBLDCT. ${ }^{[13]}$ In one study, Baur-Melnyk et al. evaluated $41 \mathrm{MM}$ patients with WBMRI and WBMDCT. WBMRI was better in the detection of the number of lesions as well as the severity of involvement. WBMDCT under staged 11 patients compared with MRI. ${ }^{[15]}$

\section{Evaluation of response to therapy in MM}

MRI is also helpful in the evaluation of response to therapy. A complete response (CR) on MRI includes complete disappearance of the marrow abnormalities and partial remission (PR) would be a change from diffuse to the focal or variegated pattern. ${ }^{[31]}$ The signal intensity reduction on T2-weighted images and resolution of enhancement of lesions, if previously present, will suggest a response to therapy. Sometimes FLs may persist or become more intense which could be due to inflammation 
or treatment-induced necrosis. ${ }^{[32,33]}$ However, there are certain limitations as it takes about 9-12 months for lesions to resolve. The patient may have received granulocyte colony-stimulating factor (GCSF) during autologous stem cell transplant (ASCT) resulting in some changes in BM which can be difficult to differentiate from the disease. In such cases, MRI should be done at least 1 month after ASCT. ${ }^{[34,35]}$

\section{Prognostic significance}

MM patients with the absence of BM abnormalities on MRI have better survival than those with abnormal findings on MRI. ${ }^{[36]}$ Furthermore, the presence of $>7$ FLs on MRI is associated with shorter survival and resolution of FLs on post-treatment MRI indicates an improved outcome. ${ }^{[30]}$

In summary, WBMRI is an ideal first-line imaging tool to assess focal and diffuse disease; however, WBLDCT or plain radiography may be used as an adjunct to MRI to detect bony destruction and make an assessment of fracture risk. There is certain limiting factor [Table 3] to the use of MRI, for example, long acquisition time, patient may be claustrophobic or have metal devices in situ and the cost. In addition, gadolinium-based contrast media may lead to nephrogenic systemic fibrosis or nephrogenic fibrosing dermopathy. ${ }^{[37]}$

\section{Positron Emission Tomography (PET)-CT}

PET is a nuclear imaging technique in which positrons are used as radiolabels. The most commonly used is 18-fluorine-fluoro-deoxyglucose (FDG). It is injected into the patient who should be fasting 4-6 h pre-procedure. Blood glucose is measured before FDG is injected and the patient undergoes scanning about 60-90 min after the injection. ${ }^{[4]}$ Active lesions show FDG uptake that is greater than the background level. FDG accumulation is measured quantitatively as standardised uptake value (SUV). However, if the lesions are smaller than $5 \mathrm{~mm}$ in diameter, then FDG uptake should be considered positive regardless of SUV and lesions are indeterminate if SUV is $<2.5$ and lesion size is between 5 and $10 \mathrm{~mm} \cdot{ }^{[11,38]}$ The PET component provides information about the function of the tissues (such as blood flow, oxygen use and metabolism of glucose) and CT provides structural/anatomical images. The combination of these two modalities can characterise the abnormal lesions both morphologically (e.g., soft tissues or bony) as well as functionally (e.g., active or inactive). Moreover, with the use of PET/CT, the lesions with subtle activity or very small size can also be detected which could be missed on PET images alone. Moreover, the scanning time is shorter with PET/CT (30 min) than with PET alone (about $1 \mathrm{~h}$ ). ${ }^{[4,39,40]}$ The extent of BM infiltration and the presence of extramedullary disease are important factors in MM patients and have prognostic significance. ${ }^{[41]}$ PET/CT and MRI are very sensitive imaging modalities to detect these abnormalities [Figure 7].

\section{Role of PET/CT at diagnosis}

PET/CT provides information both about anatomy of lesions and highlights if involved area is still active

Table 3: Advantages and limitations of magnetic resonance imaging

\begin{tabular}{l|l}
\hline Advantages & Limitations \\
\hline Excellent for axial skeleton assessment & Metallic implants \\
\hline Best modality for cord compression/nerve root compression & Claustrophobia \\
\hline Excellent for detection of diffuse disease as well as focal lesions & Availability \\
\hline $\begin{array}{l}\text { Can detect complications such as amyloid deposition in heart or other tissues and avascular necrosis of } \\
\text { femur head }\end{array}$ & Cost \\
\hline No radiation involved & \\
\hline Greater sensitivity (83.3\%) than plain radiography & \\
\hline Can be used response assessment & \\
\hline Useful in SBP & \\
\hline Maybe helpful in SM/MGUS & \\
\hline SBP. Solitary bone plasmacytoma, MGUS: Monoclonal gammopathy of underdetermined significance, SM: Smouldering myeloma
\end{tabular}



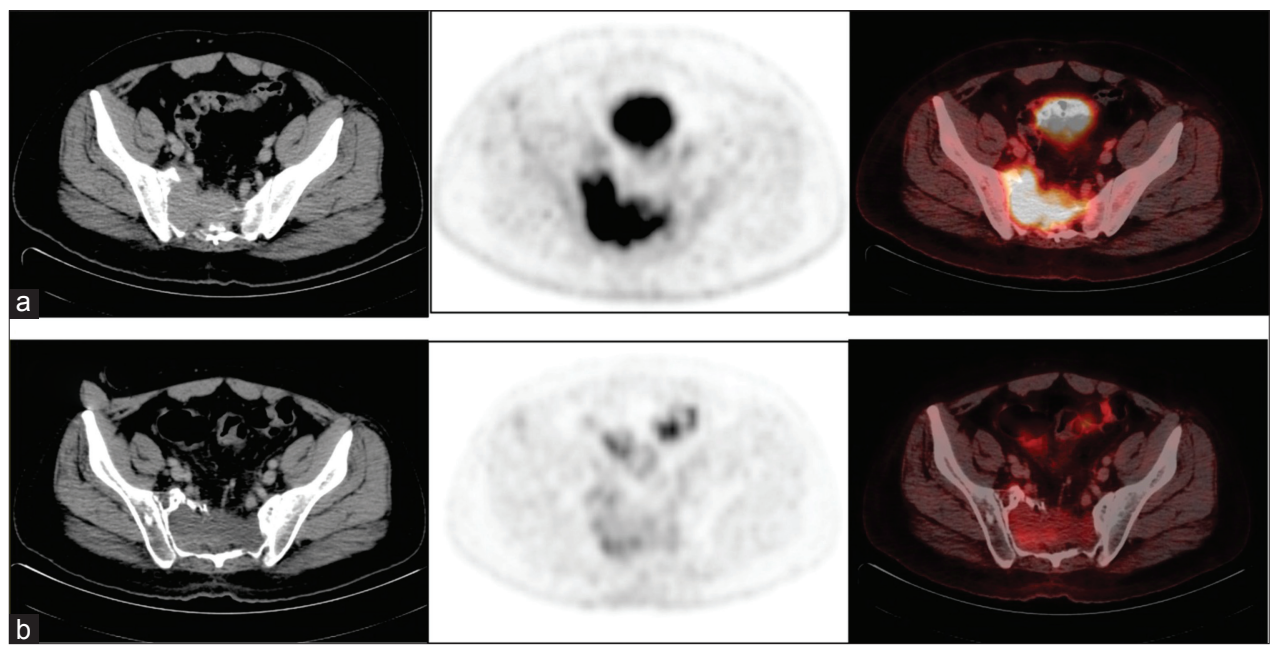

Figure 7: F18 18-fluorine-fluorodeoxyglucose positron emission tomography-computed tomography scan images of a 43-year-old patient with multiple myeloma (a) Baseline scan shows expansile lytic lesion in right sacral ala (max SUV 7.2) (b) Post cycle 4 LCD redemonstrated sacral lesion with interval reduction in uptake (max SUV 2.4 = Liver)

or inactive (depending on FDG uptake). Furthermore, $\mathrm{PET} / \mathrm{CT}$ detects osseous and extramedullary disease. In addition, there is a possibility to differentiate between diseased tissues and necrotic tissues resulting from radiation therapy. ${ }^{[40]}$

Fonti et al. reported results of $33 \mathrm{MM}$ patients who underwent PET/CT, whole body Tc-sestamibi (Tc-MIBI) and MRI of spine and pelvis. PET/CT revealed 196 FLs ( 75 were in pelvis and spine), Tc-MIBI detected 63 focal defects ( 1 in spine and 9 in pelvis) and MRI showed $51 \mathrm{FLs}$ (40 in spine and 11 in pelvis). PET/CT detected significantly more lesions compared to Tc-MIBI and MRI. Another finding was that PET/CT and MRI findings were comparable in the detection of FLs alone or combination with a diffuse pattern. However, MRI and Tc-MIBI did better in the detection of diffuse pattern. ${ }^{[39]}$ In another comparative study, Shortt et al. compared WBMRI with $\mathrm{PET} / \mathrm{CT}$ and found a positive predictive value (PPV) of $100 \%$ when both were used in combination; however, WBMRI was superior in detection of active disease with higher sensitivity ( $68 \%$ vs. $59 \%)$ and specificity $(83 \%$ vs. $75 \%){ }^{[42]}$ Likewise, Zamagni et al. evaluated $46 \mathrm{MM}$ patients with whole-body X-rays, MRI of spine-pelvis and whole-body PET/CT. In this study, PET/CT was found to be superior to plain X-rays in $46 \%$ of patients (19\% of patients were false negative on X-rays). In 35\% of cases, PET/CT was better than MRI in the detection of myelomatous deposits in the areas which were out of a field of view of MRI. Conversely, in 30\% of cases, $\mathrm{PET} / \mathrm{CT}$ did not reveal any lesions in the spine and pelvic areas where MRI revealed BM involvement, particularly in a diffuse pattern. ${ }^{[43]}$ Bredella et al. evaluated $13 \mathrm{MM}$ patient with FDG PET. In this study, 4 out of 13 patients were upstaged by FDG PET resulting in a change in future management. ${ }^{[44]}$ The sensitivity and specificity of FDG PET to detect the medullary involvement of MM was $85 \%$ and $92 \%$, respectively.

Recently, Bartel et al. demonstrated in a large group of patients that the number and FDG positivity of FLs on PET/CT were correlated with high beta-two microglobulin, C-reactive protein (CRP) and lactate dehydrogenase levels. Furthermore, the presence of $>3$ FDG-avid FLs was associated with inferior overall and event-free survival. ${ }^{[45]}$ In another prospective study $(n=43) \mathrm{PET} / \mathrm{CT}$ was positive in 38 out of 41 (sensitivity 92.7\%) known lytic lesions due to MM; furthermore, 71 additional lesions were depicted by PET/CT which were not seen on plain radiography in 14 patients which resulted in a change in management plan in about $14 \%$ of patients. The PPV for the active disease was $100 \%$ for the focal or focal/diffuse pattern and $75 \%$ for diffuse BM uptake. ${ }^{[4]}$

$\mathrm{PET} / \mathrm{CT}$ is also a useful modality in the detection of extramedullary disease and one study additional lesions were noted in up to $30 \%$ of patients who were diagnosed as SBP by MRI. PET/CT is an ideal modality to monitor 
nonsecretory MM patients and to detect osteonecrosis of jaw related to bisphosphonate therapy. ${ }^{[11,47,48]}$

\section{Role of PET/CT in the assessment of response to therapy}

PET/CT is not only capable of detecting bone lesions and extramedullary disease but also has the ability to distinguish active disease from post-therapy necrotic tissue. It is well known that bone defects due to MM rarely heal; thus, conventional radiography and CT may not be of much help in assessing the disease response post-therapy. The abnormalities noticed on MRI do change or disappear in responding patients; however, it takes about 9-12 months for these changes to be appreciated on MRI. ${ }^{[35,49]}$ In contrast, the changes in PET appear within a few hours to 3-4 weeks of effective treatment, making it the investigation of choice for restaging. The persistence of FDG avid lesions on PET/CT post-transplant is a poor prognostic factor and correlates with early relapse (within 6 months period). ${ }^{[35]}$ In one study, 10 patients had posttreatment PET/CT scans and nine of them were negative and one was positive for the active disease. None of PET/CT negative patients showed any abnormality on MRI and CT. These patients were followed up clinically for at least 6 months and eight of them remained in remission. ${ }^{[44]}$ Zamagni et al. evaluated 23 patients 3 months post autologous transplantation with PET/CT and MRI. 15 patients (65\%) showed normalisation of $\mathrm{PET} / \mathrm{CT}$ scans and in 12 of these patients, there was $\geq 90 \%$ reduction in the level of M-protein. On the other hand, of these 15 patients, MRI pattern was normal in eight while in 7 patients MRI was either unchanged or revealed a low number of lesions. ${ }^{[43]}$ The presence of residual FDG activity after induction therapy predicts poor outcome. Bartel et al. reported that complete FDG suppression before transplantation correlates with superior overall survival (OS) and event-free survival (EFS). At 30 months, there was a significant difference in OS $(92 \%$ vs. $71 \%$ ) and EFS (89\% vs. 63\%) between the patients with negative scans and those who did not achieve $100 \%$ suppression, respectively. ${ }^{[45]}$

In brief, PET/CT is an ideal imaging modality to scan whole-body in one procedure and in a reasonable time frame. It provides both functional and morphological characteristics of the lesion. It is most sensitive modality in the evaluation of treatment response, particularly in non-secretary MM. PET/CT is very useful in the detection of extramedullary disease and complications of MM (e.g., infections and ONJ). There are certain pitfalls of PET/CT which should be kept in mind while making an assessment. For example, there is high radiation exposure and smaller lesions may be missed due to limited PET resolution. There is a risk of false negative results in cases of diffuse disease involving the spine and pelvis. Inflammation, infections and GCSF treatment may increase FDG activity while radiotherapy may decrease FDG uptake on PET/CT [Table 4].

\section{9-m МIB}

99-m Tc-labelled 2-methoxyisobutylisonitrile (MIBI) is a radiopharmaceutical which emits X-rays and has a predilection to accumulate in tissues with high cell density and mitochondrial activation which could be found in malignant lesions. MIBI actively concentrated in myeloma tissues and bone lesions and is more sensitive

Table 4: Advantages and limitations of positron emission tomography-computed tomography

\begin{tabular}{l|l}
\hline Advantages & Limitations \\
\hline Ability to detect both focal and diffuse disease & High radiation exposure \\
\hline Differentiation of active and non-active lesions & Subcentimeter lesions may be missed on PET \\
\hline Ideal for response evaluation in non-secretory myeloma & Inflammation, infection, GCSF and radiotherapy can affect FDG uptake \\
\hline Detection of osteonecrosis of jaw & Availability \\
\hline $\begin{array}{l}\text { Prognostic significance (e.g., three or more lesions } \\
\text { confers poor prognostic factor and the presence of FDG } \\
\text { avidity post induction correlates with poor outcome) }\end{array}$ & \\
\hline Detection of extramedullary disease & \\
\hline
\end{tabular}

FDG: 2-fluoro-2-deoxy-D-glucose, GCSF: Granulocyte colony-stimulating factor, PET: Positron emission tomography 
to plain radiography. ${ }^{[11,50]}$ It has been demonstrated that MIBI localises inside the plasma cells. ${ }^{[51]}$ There are different patterns of MIBI uptake in MM; physiological, focal, diffuse and extramedullary uptake. Focal uptake indicates active myeloma, but diffuse uptake without focal positivity does not support active myeloma. ${ }^{[52,53]}$ Mele et al. evaluated 397 cases of MM with MIBI and conventional X-rays. In a series of 229 MIBI scans done at diagnosis, 146 (64\%) were positive and 81 cases were discordant with X-ray results. The sensitivity was higher (77\%) with MIBI compared to X-rays (45\%). In addition, MIBI results were positively correlated with CRP, BM infiltration and bone pain. In 168 follow-up scans, MIBI presented high specificity in patients with CR and correlated with response to therapy. ${ }^{[50]}$ In another study, MIBI was found very effective in determining the active myeloma disease both at diagnosis and relapse. ${ }^{[54]}$ It has been reported that MIBI has the ability to detect active lesions in non-secretory MM and MIBI results directly correlate with clinical outcome after ASCT. ${ }^{[53,55]}$

However, MIBI is inferior to PET/CT and MRI in the detection of myelomatous involvement. In one study, FDG-PET was better than MIBI in the detection of skeletal lesions (93.3\% vs. 80\%), soft tissue lesions (68.4\% vs. $89.5 \%$ ) and BM infiltration (100\% vs. $80 \%) .{ }^{[56]}$ Fonti et al. reported a comparison of PET/CT, MIBI and MRI. In this study, PET/CT was superior to MIBI in the detection of FLs but inferior in cases of the diffuse disease. Likewise, MIBI was inferior to MRI in the detection of disease, particularly in spine and pelvis. ${ }^{[39]}$ In brief, MIBI is a useful whole-body imaging modality in MM particularly if PET/CT and MRI are not available.

\section{Bone Scan (Bone Scintigraphy)}

Bone scan is a whole-body imaging to detect metabolic activity in the entire skeleton using 99m Tc-diphosphonate as a radiopharmaceutical. The mechanism of uptake is directly correlated with blood flow and osteoblastic activity of the lesion. In MM, the bony lesions are due to increased osteoclastic activity and suppressed or absent osteoblastic function; thus, a bone scan is not very useful in these patients. Ludwig et al. reported a comparison of bone scan and plain radiography in $41 \mathrm{MM}$ patients. In this study, plain radiography was superior to a bone scan. Plain radiography detected 97 myeloma-related lesions compared to only 16 with bone scintigraphy, giving a sensitivity of $91 \%$ and $46 \%$, respectively. ${ }^{[57]}$ In another study, $27 \mathrm{MM}$ patients were monitored for about 5 years with bone scintigraphy and SKS every 6 months. The SKS revealed myeloma-related lesions with high sensitivity and specificity at the diagnosis, in follow-up cases and the assessment of bone pain than bone scintigraphy. ${ }^{[58]}$ To sum up, a bone scan is not routinely used in MM, the major reason being the primary dysfunction of osteoblasts in MM and 99-m Tc-diphosphonate uptake is dependent on osteoblastic activity. However, a bone scan can be helpful in the detection of fracture sites.

\section{Dual-energy X-ray Absorptiometry (DXA)}

DXA scan is a method of choice for the diagnosis of osteoporosis. There is an increased risk of early vertebral fracture in MM patients who have reduced lumbar spine bone mineral density (BMD). This makes DXA scan an important test to consider in these patients particularly when there is no focal lytic lesion. ${ }^{[47,59]}$ However, DXA may not be able to differentiate between myelomarelated osteoporosis from other causes of osteoporosis. Abildgaard et al. evaluated 34 newly diagnosed MM patients with DEXA and demonstrated that osteopenia of spine correlates with increased risk of vertebral fracture. DEXA may be useful to identify high-risk patients who may need intensive chemotherapy or bisphosphonate treatment. ${ }^{[60]}$ In one study, $66 \mathrm{MM}$ patients were monitored with DEXA scan to assess BMD. Patients were treated either with conventional therapy or intensive therapy with peripheral blood stem cell transplantation. After intensive therapy, the increase of lumbar spine BMD was higher in men than women. There was a correlation with changes in BMD and M-protein in urine or serum. ${ }^{[61]}$ The presence of lytic lesions, vertebral collapse and spinal osteophytes may cause difficulties in some patients to do DEXA scan. In selected cases femoral and radial BMD can be relied on.

\section{Conclusion}

Due to the complex nature of the human skeleton, a single imaging technique cannot provide a comprehensive picture of the whole body. All imaging modalities have some advantages and disadvantages. Plain radiography is still accepted as a primary investigation for the screening of MM patients. However, WBMRI, CT and PET/CT scans have replaced plain radiography in some institutions 
and should be considered depending on availability. The cost of these imaging tools and the availability of trained professionals are major obstacles to using them widely. WBMRI should be performed in the cases of MM with normal SKS at diagnosis, particularly in patients with SBP. An urgent MRI is indicated in suspected spinal cord compression. $\mathrm{CT}$ of the spine may be an alternative if MRI is not available. CT may also be considered for further evaluation of lesions which are inconclusive on MRI to detect structural damage of the bone and extramedullary masses. PET/CT is most sensitive in diagnosing extramedullary disease and is also considered ideal in patients with non-secretory MM. In addition, $\mathrm{PET} / \mathrm{CT}$ appears to be better in the assessment of response to therapy than other available techniques and should be considered. Based on available data, the MIBI, bone and DEXA scans do not have a significant role in the management of MM and are not used routinely.

\section{Acknowledgment}

We are grateful to Dr. Humayun Bashir and Dr. Amjad Iqbal for providing PET and MR images shown in this article.

\section{Conflict of Interest}

The authors declare that they have no conflict of interest.

\section{References}

1. Kyle RA, Rajkumar SV. Multiple myeloma. N Engl J Med 2004;351:1860-73.

2. Angtuaco EJ, Fassas AB, Walker R, et al. Multiple myeloma: Clinical review and diagnostic imaging. Radiology 2004;231:11-23.

3. Kyle RA, Gertz MA, Witzig TE, et al. Review of 1027 patients with newly diagnosed multiple myeloma. Mayo Clin Proc 2003;78:21-33.

4. D'Sa S, Abildgaard N, Tighe J, et al. Guidelines for the use of imaging in the management of myeloma. Br J Haematol 2007;137:49-63.

5. Kyle RA, Rajkumar SV. Criteria for diagnosis, staging, risk stratification and response assessment of multiple myeloma. Leukemia 2009;23:3-9.

6. Roodman GD. Pathogenesis of myeloma bone disease. Blood Cells Mol Dis 2004;32:290-2.

7. Collins CD. Multiple myeloma. Cancer Imaging 2004;4 Spec No A: S47-53.

8. Winterbottom AP, Shaw AS. Imaging patients with myeloma. Clin Radiol 2009;64:1-1.
9. Zangari M, Esseltine D, Lee CK, et al. Response to bortezomib is associated to osteoblastic activation in patients with multiple myeloma. Br J Haematol 2005;131:71-3.

10. Mulligan ME. Skeletal abnormalities in multiple myeloma. Radiology 2005;234:313-4.

11. Dimopoulos M, Terpos E, Comenzo RL, et al. International myeloma working group consensus statement and guidelines regarding the current role of imaging techniques in the diagnosis and monitoring of multiple myeloma. Leukemia 2009;23:1545-56.

12. Mahnken AH, Wildberger JE, Gehbauer G, et al. Multidetector CT of the spine in multiple myeloma: Comparison with MR imaging and radiography. AJR Am J Roentgenol 2002;178:1429-36.

13. Gleeson TG, Moriarty J, Shortt CP, et al. Accuracy of whole-body low-dose multidetector CT (WBLDCT) versus skeletal survey in the detection of myelomatous lesions, and correlation of disease distribution with whole-body MRI (WBMRI). Skeletal Radiol 2009;38:225-36.

14. Hur J, Yoon CS, Ryu YH, et al. Efficacy of multidetector row computed tomography of the spine in patients with multiple myeloma: Comparison with magnetic resonance imaging and fluorodeoxyglucose-positron emission tomography. J Comput Assist Tomogr 2007;31:342-7.

15. Baur-Melnyk A, Buhmann S, Becker C, et al. Whole-body MRI versus whole-body MDCT for staging of multiple myeloma. AJR Am J Roentgenol 2008;190:1097-104.

16. Hanrahan CJ, Christensen CR, et al. Current concepts in the evaluation of multiple myeloma with MR imaging and FDG PET/CT. Radiographics 2010;30:127-42.

17. Moulopoulos LA, Varma DG, Dimopoulos MA, et al. Multiple myeloma: Spinal MR imaging in patients with untreated newly diagnosed disease. Radiology 1992;185:833-40.

18. Stäbler A, Baur A, Bartl R, et al. Contrast enhancement and quantitative signal analysis in MR imaging of multiple myeloma: Assessment of focal and diffuse growth patterns in marrow correlated with biopsies and survival rates. AJR Am J Roentgenol 1996;167:1029-36.

19. Libshitz HI, Malthouse SR, Cunningham D, et al. Multiple myeloma: Appearance at MR imaging. Radiology 1992;182: 833-7.

20. Lütje S, de Rooy JW, Croockewit S, et al. Role of radiography, MRI and FDG-PET/CT in diagnosing, staging and therapeutical evaluation of patients with multiple myeloma. Ann Hematol 2009;88:1161-8.

21. Lecouvet FE, Malghem J, Michaux L, et al. Vertebral compression fractures in multiple myeloma. Part II. Assessment of fracture risk with MR imaging of spinal bone marrow. Radiology 1997;204:201-5.

22. Lafforgue P, Dahan E, Chagnaud C, et al. Early-stage avascular necrosis of the femoral head: MR imaging for prognosis in 31 cases with at least 2 years of follow-up. Radiology 1993;187:199-204.

23. Moulopoulos LA, Dimopoulos MA, Weber D, et al. 
Magnetic resonance imaging in the staging of solitary plasmacytoma of bone. J Clin Oncol 1993;11:1311-5.

24. Moulopoulos LA, Dimopoulos MA, Smith TL, et al. Prognostic significance of magnetic resonance imaging in patients with asymptomatic multiple myeloma. J Clin Oncol 1995;13: 251-6.

25. Rajkumar SV, Dimopoulos MA, Palumbo A, et al. International myeloma working group updated criteria for the diagnosis of multiple myeloma. Lancet Oncol 2014; 15:e538-48.

26. Bellaïche L, Laredo JD, Lioté F, et al. Magnetic resonance appearance of monoclonal gammopathies of unknown significance and multiple myeloma. The GRI study group. Spine (Phila Pa 1976) 1997;22:2551-7.

27. Berg BC, Michaux L, Lecouvet FE, et al. Nonmyelomatous monoclonal gammopathy: Correlation of bone marrow MR images with laboratory findings and spontaneous clinical outcome. Radiology 1997;202:247-51.

28. Laroche M, Assoun J, Sixou L, et al. Comparison of MRI and computed tomography in the various stages of plasma cell disorders: Correlations with biological and histological findings. Myélome-midi-pyrénées group. Clin Exp Rheumatol 1996;14:171-6.

29. Ghanem N, Lohrmann C, Engelhardt M, et al. Wholebody MRI in the detection of bone marrow infiltration in patients with plasma cell neoplasms in comparison to the radiological skeletal survey. Eur Radiol 2006;16:1005-14.

30. Walker R, Barlogie B, Haessler J, et al. Magnetic resonance imaging in multiple myeloma: Diagnostic and clinical implications. J Clin Oncol 2007;25:1121-8.

31. Moulopoulos LA, Dimopoulos MA, Alexanian R, et al. Multiple myeloma: MR patterns of response to treatment. Radiology 1994;193:441-6.

32. Baur-Melnyk A, Buhmann S, Dürr HR, et al. Role of MRI for the diagnosis and prognosis of multiple myeloma. Eur J Radiol 2005;55:56-63.

33. Rahmouni A, Divine M, Mathieu D, et al. Detection of multiple myeloma involving the spine: Efficacy of fatsuppression and contrast-enhanced MR imaging. AJR Am J Roentgenol 1993;160:1049-52.

34. Hartman RP, Sundaram M, Okuno SH, et al. Effect of granulocyte-stimulating factors on marrow of adult patients with musculoskeletal malignancies: Incidence and MRI findings. AJR Am J Roentgenol 2004;183:645-53.

35. Durie BG. The role of anatomic and functional staging in myeloma: Description of durie/Salmon plus staging system. Eur J Cancer 2006;42:1539-43.

36. Lecouvet FE, Berg BC, Michaux L, et al. Stage III multiple myeloma: Clinical and prognostic value of spinal bone marrow MR imaging. Radiology 1998;209:653-60.

37. Marckmann P, Skov L, Rossen K, et al. Nephrogenic systemic fibrosis: Suspected causative role of gadodiamide used for contrast-enhanced magnetic resonance imaging. J Am Soc Nephrol 2006;17:2359-62.

38. Sager S, Ergül N, Ciftci H, et al. The value of FDG PET/CT in the initial staging and bone marrow involvement of patients with multiple myeloma. Skeletal Radiol 2011;40: 843-7.

39. Fonti R, Salvatore B, Quarantelli M, et al. 18F-FDG PET/CT, 99mTc-MIBI, and MRI in evaluation of patients with multiple myeloma. J Nucl Med 2008;49:195-200.

40. Nanni C, Zamagni E, Farsad M, et al. Role of 18F-FDG $\mathrm{PET} / \mathrm{CT}$ in the assessment of bone involvement in newly diagnosed multiple myeloma: Preliminary results. Eur J Nucl Med Mol Imaging 2006;33:525-31.

41. Durie BG, Waxman AD, D'Agnolo A, et al. Whole-body (18)F-FDG PET identifies high-risk myeloma. J Nucl Med 2002;43:1457-63.

42. Shortt CP, Gleeson TG, Breen KA, et al. Whole-body MRI versus PET in assessment of multiple myeloma disease activity. AJR Am J Roentgenol 2009;192:980-6.

43. Zamagni E, Nanni C, Patriarca F, et al. A prospective comparison of $18 \mathrm{~F}$-fluorodeoxyglucose positron emission tomography-computed tomography, magnetic resonance imaging and whole-body planar radiographs in the assessment of bone disease in newly diagnosed multiple myeloma. Haematologica 2007;92:50-5.

44. Bredella MA, Steinbach L, Caputo G, et al. Value of FDG PET in the assessment of patients with multiple myeloma. AJR Am J Roentgenol 2005;184:1199-204.

45. Bartel TB, Haessler J, Brown TL, et al. F18fluorodeoxyglucose positron emission tomography in the context of other imaging techniques and prognostic factors in multiple myeloma. Blood 2009;114:2068-76.

46. Schirrmeister H, Bommer $\mathrm{M}$, et al. Initial results in the assessment of multiple myeloma using 18F-FDG PET. Eur J Nucl Med Mol Imaging 2002;29:361-6.

47. Mulligan ME, Badros AZ. PET/CT and MR imaging in myeloma. Skeletal Radiol 2007;36:5-16.

48. Catalano L, Del Vecchio S, Petruzziello F, et al. Sestamibi and FDG-PET scans to support diagnosis of jaw osteonecrosis. Ann Hematol 2007;86:415-23.

49. Wiesenthal AA, Nguyen BD. F-18 FDG PET/CT staging of multiple myeloma with diffuse osseous and extramedullary lesions. Clin Nucl Med 2007;32:797-801.

50. Mele A, Offidani M, Visani G, et al. Technetium-99m sestamibi scintigraphy is sensitive and specific for the staging and the follow-up of patients with multiple myeloma: A multicentre study on 397 scans. Br J Haematol 2007;136: 729-35.

51. Ak I, Aslan V, Vardareli E, et al. Tc-99m methoxyisobutylisonitrile bone marrow imaging for predicting the levels of myeloma cells in bone marrow in multiple myeloma: Correlation with CD38/CD138 expressing myeloma cells. Ann Hematol 2003;82:88-92.

52. Nandurkar D, Kalff V, Turlakow A, et al. Focal MIBI uptake is a better indicator of active myeloma than diffuse uptake. Eur J Haematol 2006;76:141-6.

53. Svaldi M, Tappa C, Gebert U, et al. Technetium-99msestamibi scintigraphy: An alternative approach for 
diagnosis and follow-up of active myeloma lesions after high-dose chemotherapy and autologous stem cell transplantation. Ann Hematol 2001;80:393-7.

54. Fallahi B, Saghari M, Esfahani AF, et al. The value of $99 \mathrm{mTc}-\mathrm{MIBI}$ whole body scintigraphy in active and in remission multiple myeloma. Hell J Nucl Med 2005;8:165-8.

55. Catalano L, Andretta C, Pace L, et al. Tc99m-sestaMIBI uptake in nonsecretory multiple myeloma. Hematology 2005;10: 335-8.

56. Hung G, Tsai C, Tsai S, et al. Comparison of Tc-99m Sestamibi and F-18 FDG-PET in the assessment of multiple myeloma. Anticancer Res 2005;25:4737-41.

57. Ludwig H, Kumpan W, Sinzinger H. Radiography and bone scintigraphy in multiple myeloma; a comparative analysis.
Br Radiol 1982;55:173-81.

58. Wahner HW, Kyle RA, Beabout JW. Scintigraphic evaluation of the skeleton in multiple myeloma. Mayo Clin Proc 1980;55:739-46.

59. Abildgaard N, Brixen K, Eriksen E, et al. Sequential analysis of biochemical markers of bone resorption and bone densitometry in multiple myeloma. Haematologica 2004;89: 567-77.

60. Abildgaard N, Brixen K, Kristensen JE, et al. Assessment of bone involvement in patients with multiple myeloma using bone densitometry. Eur J Haematol 1996;57:370-6.

61. Mariette X, Bergot C, Ravaud P, et al. Evolution of bone densitometry in patients with myeloma treated with conventional or intensive therapy. Cancer 1995;76:1559-63. 